\title{
Techniques of Vibration Signature Analysis
}

\author{
Sumit Kumar Sar ${ }^{1}$, Dr. Ramesh Kumar ${ }^{2}$ \\ P.hD Scholar, Department of Computer Science \& Engineering, BIT, Durg, India ${ }^{1}$ \\ Department of Computer Science \& Engineering, BIT, Durg, India ${ }^{2}$
}

\begin{abstract}
This paper presents recent developments of techniques, based on vibration signature analysis to detect the fault in a machine. This paper shows vibration signature analysis implemented to detect fault in various machineries such as gear, rolling element bearing, journal bearing, induction motor, centrifugal pump and beam. The authors do not intend to provide an exhaustive discussion about machine faults but only detailed overview of rotating parts vulnerable to fault. In addition, advantages of advanced vibration signature analysis techniques over traditional one have also been discussed.
\end{abstract}

Keywords: Machine fault, condition monitoring, vibration signature, preventive maintenance.

\section{INTRODUCTION}

The main objective of maintenance department is to keep 3. machinery and plant equipments in good operating piece of equipment, machine, or system to the specified condition that prevents failure and production loss [1]. operable condition to achieve its maximum useful life. It Monitoring of a rotating machine has been a challenging includes corrective task for engineers and researchers especially in industries. maintenance.

There are many monitoring methods available which require expensive sensors and specialized tools. There was a need felt for a cheaper, yet more precise technique for identifying and preventing rather than curing problems resulting in shutdowns/breakdowns [2]. Breakdown Maintenance costs can be drastically cut with the help of Condition Based Monitoring (CBM). The idea of CBM is to perform maintenance only when there is an actual requirement, while ensuring safety, apparatus reliability and decrease in ${ }^{1}$ total cost [3][4]. Vibration analysis is a powerful process of performing all of the following:

a) Current machine Status.

b) Diagnosing defects related with operational machines.

c) Monitoring the overall condition of machines.[5] A complete vibration analysis consists of the following three components:

a) Exact vibration determination

b) Bearing condition determination

c) FFT analysis

This paper is divided into different sections, each dealing with various aspects of the subject. It begins with maintenance of machine followed by types of monitoring; vibration analysis uses in different machineries till date and general overview of the advanced techniques of signature analysis.

\section{MAINTENANCE PROCEDURE}

\section{Definition:}

1. Care or upkeep of machines is known as maintenance.

2. Activities required or undertaken to conserve as nearly, and as long as possible the original condition of an asset or resource while compensating for normal wear and tear.
Maintenance requires knowledge of science, a thought process and an artistic fervor. Maintenance requires understanding the real as well as literal meaning of the word maintenance. Effective maintenance is 'Just -InTime'. [6]

Types of Maintenance

A. Breakdown maintenance

B. Preventive maintenance

a. Periodic maintenance

b. Predictive Maintenance

C. Corrective maintenance

\section{III.MONITORING TECHNIQUES}

Breakdown monitoring of machines is no longer a preferred method used in industries. Preventive and non destructive monitoring techniques are, thus, becoming more and more popular. The main benefits of such techniques are:

- $\quad$ Easy and early identification of fault.

Almost total prevention of breakdown.

Lesser loss of production time.

Optimum utilization of machine life period.

Condition-Based Monitoring has emerged as a precise and easily applicable technique of the others.

\section{A. Conditioning-Based Monitoring}

Def: Condition-Based Monitoring may be defined as the process of monitoring a parameter of condition of a machine, any impending technical failure or fault may become apparent. Predictive maintenance renders maintenance activities frequent and scheduled, such that any failure may be avoided at an early stage [7][8][9][10][11][12][13]. The following are some CBM techniques that have been developed in the recent past: 

a) Lubrication Analysis
b) Shock impact analysis
c) Low frequency monitoring
d) Higher-order spectral techniques
e) Wear debris analysis
f) Ultra Sound Analysis
g) Temperature Analysis

\section{IV.VIBRATION SIGNATURE ANALYSIS}

The vibration signature of a machine is the characteristic pattern of vibration it generates while it is in operation [14]. The actual signal from a vibration transducer can be considered the signature, but the spectrum of the vibration signal is usually referred to as the signature. Effective vibration analysis starts with obtaining an accurate signal from standard vibration transducer with the help of an accelerometer. The analog signal is then converted in to digital signal using analog to digital converter. The digital signals can be processed directly or can be processed using some formula depending upon the need of the user [15].

\section{A. Visual Analysis}

Visual inspection is the most basic and primary form of condition monitoring. Visual inspection is done through experienced operators and maintainers. With the help of visual inspection, detection of fault such as crack, leak, corrosion, cavitations etc can be identify in the machine before failure. Visual inspection is the most inexpensive conditioning monitoring.

\section{B. Nondestructive testing}

Non destructive testing (NDT) is an analysis technique used in science and industry to evaluate the properties of a material, component or system without causing damage [16]. The

terms Nondestructive examination (NDE), Nondestructive inspection (NDI) and Nondestructive evaluation (NDE) are also commonly used to describe this technology [17].Because NDT does not permanently alter the machine being inspected, it is a highly-valuable technique that can save both money and time in product evaluation, troubleshooting, and research.

\section{Motor Current Signature Analysis}

Motor Current Signature Analysis (MCSA) is a technique used to determine the operating condition of $\mathrm{AC}$ induction motors without interrupting production by analyzing current and voltage supplied to an electric motor with the help of specific patterns and protocols. MCSA along with vibration and thermal analysis is used to make machinery diagnostic decisions. MCSA operates on the principle that induction motor circuit is effectively a transducer. By clamping a Hall Effect Current sensor on either the primary or secondary circuit, fluctuations in motor current can be observed [18].

\section{USES IN VARIOUS MACHINERIES TILL DATE}

\section{A. Gear}

According to Enayet B. Halim, Sirish L. Shah, Ming J. Zuo and M. A. A. Shoukat Choudhury, a fault in the gears of a gearbox can be detected by analysis of its vibration signatures using different signal processing techniques [19]. They propose a new technique combining the Time Domain average and wavelet transformation. It extracts the periodic waveforms at different scales from noisy vibration signals and detects both local and distributed faults simultaneously [20].

\section{B. Rotor}

According to Eduardo Rubio and Juan C. Jáuregui There are some methods available for rotor friction to analyze the vibrations signals for preserving the time and scale information. The conventional processing techniques for rotor friction such as FFT cannot process accurately. The analysis of output data gives an idea about that light rubbing results in a vibration characterized by a good frequency spectrum, and the rigorous rubbing is effectively described as an impact. Impacts could be identified and localized with wavelets in the upperfrequency sub bands [21].

\section{Cantilever shaft}

According to D. Parhi, R. K. Behera, R. K. Behera analysis of vibration signature is applied to detect fault in cantilever shaft that if crack is present in the shaft, the natural frequencies of the shaft structure is changed and the change in frequency is totally depend upon the crack location and its amount. The neural network based system is developed to calculate the exact location and the amount of crack by using natural frequencies as input [22].

\section{Rolling Elements Bearing}

According to Jason R. Stack, Thomas G. Habetler, and Ronald G. Harley [23] [47] analysis of Vibration signature is applied to detect fault in bearings in three ways:

1. Fault may be in Inner-race, as their fault-index counts are greater than healthy bearings.

2. Fault may be in Bearings, as their fault-index counts are low (between 0-5).

3. Fault may be in rolling-element defects.

\section{E. Journal bearing}

According to Bo Li, Mo-Yuen Chow, Yodyium Tipsuwan, and James C. Hung vibration signature analysis to detect fault, is applied on bearing in both time domain and frequency domain they detect the motor bearing fault using neural networks algorithm [24].

\section{F. Induction motor}

According to Neelam Mehala, and Ratna Dahiya, Motor current signature analysis can reliably analyze rotor cage problems in induction motor drives. Conventional methods of measurements give fake alarms or incorrect analysis of healthy machines because of current frequency components being there in the stator current. Advanced tools are available to check these components and hence MCSA is more powerful, free from error and robust technology. They present the case studies which show the ability to divide current components based on theoretical analysis. The case studies also show that MCSA can successfully identify cracked rotors and other faults [25]. 


\section{G. Beam}

According to D.R.K. Parhi and A.K. Dash [26] Vibration analysis for detection of cracks in a beam is good enough as compared to conventional methods. Vibration analysis can find out the location and size of the cracks from the cracked beam structure. The crack in the beam gives rise to a decrease in natural frequencies and the change in the shapes. Thus, it may be possible to calculate approximate location and size of the cracks in the beam by analyze the changed vibration parameters. Conclusions obtained from the various analyses are described below.

- They present a simple and efficient method to detect multiple cracks in a beam from the analysis of the vibration signatures

- $\quad$ The detection of crack locations and sizes using Finite Element Method from theoretical and experimental analysis

- The method can be used to model any engineering structures and online condition monitoring of damaged structures.

\section{H. Centrifugal pump}

According to M.R. Nasiri, M.J. Mahjoob, H. VahidAlizadeh analysis of Vibration signature is applied to detect fault (cavitations) in a centrifugal pump using a neural network system. The features extracted from vibration signals are used as inputs to the neural network. The output data of the system is represent 0 for normal condition, 0.5 for developed cavitations and 1 for fully developed cavitations [27].

\section{Cracked rotor in liquid}

According to R. K. BEHERA, D. R. K. PARHI, and S. K. SAHU [28] that the effect of crack depths and locations on modal properties of the rotor shaft are analyzed using vibration signature. The changes in the natural frequencies and amplitudes of vibration are detected due to noticeable change in the crack location and depth of the cracks. The existence of cracks in viscous medium can affect the amplitudes of vibration. The following conclusions can be drawn from the analysis:

1) The natural frequencies decrease due to increase in the depth of the cracks.

2) The critical speed decreases due to existence of a crack.

3) The damping coefficient increases due to the decrease in critical speed.

\section{J. Cooling pump}

According to S. Devi, Dr. L. Siva Kumar, N.R.Shanker and K.Prabakaran [29], that the standard FFT values for the vibration and acoustic signals are used to determine the status of the machine using analyzed samples fetched from the machines, The Status of the machines is divided into three states.

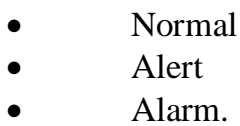

Copyright to IJARCCE
The state monitoring of the machines can be done effectively using the vibrations of the machine as well as the sound signals. The acoustic and vibration signals both are used for condition monitoring; it is observed that the acoustic analysis cost is not as much of the vibration analysis [45].

\section{VI.ADVANCED TECHNIQUES FOR VIBRATION SIGNATURE ANALYSIS}

Conventional techniques of signal processing have become obsolete these days as newer, more advanced and more accurate techniques have taken over. Availability of better sensors has facilitated a huge quantity of data, that the traditional techniques were unable to process accurately. This short coming was overcome by the advent of an algorithm based technique, namely Neural Networks. Neural networks are processors that have the ability to acquire knowledge through a learning process when there is lots of training data available and then store this knowledge. Condition monitoring process has some notable imprecision. Also $\mathrm{NN}$ is adaptable, whereas intelligent systems have strict rules. The fuzzy logic was developed as a means for representing, manipulating, and utilizing uncertain information. Fuzzy logic is capable of relating input to output in linguistic terms by simplifying knowledge acquisition and representation [46]. It has an edge over its contemporary methods as it can achieve a steady state in a shorter time interval. Development of a hybrid system by combining NN and Fuzzy logic has resulted in overcoming of their individual drawbacks in the form of FNN systems. Wavelet Transform analysis is a mathematical tool and algorithm that reduces a Time domain signal into its component frequency domain signals. Recent systems have relied on artificial intelligence techniques as well as fuzzy logic system to strengthen the robustness of diagnostics systems. Four artificial techniques have been widely applied as expert system, neural networks, fuzzy logic, and model-based systems [30] [48].

\section{CONCLUSION}

This review paper presents a brief overview of recently developed techniques for detecting faults using vibration signature analysis. Since industrial revolution, maximization of production has been the focus of not only industrialists, but also scientists and researchers such as Satish and Sharma [31], Duraisamy et al. [32], M. Samhouri et al. [33], Travers Snyman et al. [33], Bo Li et al [24], I. Lasurt et al. [10], Wang Huaying and Wang Guangjian [35], Lin Niu [36], Wang Yuguo et al. [37], Jun and Lias. [47], L. Navarro [38], Qun Ren et al. [39], Deng Lin-feng and Zhao Rong-zhen [40], M.R. Nasiri et al. [27], D. M. Yang [41], S.K. Panigrahi et al. [42], Sun, Chen, Zhang, and Xi (2004) [43], Vasile Palade et al [44], who have been engaged in the conceptualization of more efficient machine monitoring systems, since any loss of production time due to failure of machines is becoming increasingly unacceptable. Various system and procedures have evolved with time due to rapid technological developments, such as computer hardware, electronic 
sensors, transducers, etc. which help in improved monitoring of machines. Also analytical systems have been developed with the help of Neural Networks, fuzzy logic, wavelet transformations and their hybrids, i.e. fuzzy neural networks, wavelet transformation and fuzzy logic. This has and will result in better functioning of machines, longer and healthier life and a drastic reduction in loss of production.

Various vibration signature analysis techniques that are prevalent have been presented along with their applications on different rotating machinery parts such as shafts, beams, bearings, rotors, gears, pumps, etc. Also, latest advancements in vibration signature analysis techniques have been discussed. This paper has tried to present the current monitoring and vibration signature analysis scenario.

\section{ACKNOWLEDGMENT}

This work is supported by Department of Computer Science and Engineering, Bhilai Institute of Technology, Durg and Dr. Ramesh Kumar

\section{REFERENCES}

[1] H. P. Bloch and F. K. Geitner, Machinery Failure Analysis and Troubleshooting, chapter 5, Gulf Publishing Company, Houston, Tex, USA, 1983.

[2] Derya Sevim Korkut, Ilter Bekar and Ertugrul Oztekin, Application of preventive maintenance planning in a parquet enterprise, African Journal of Biotechnology Vol. 8 (8), pp. 1665-1671, 20 April, 2009.

[3] S.K.Sethiya, Condition Based Monitoring.

[4] IAEA, Implementation Strategies and Tools for Condition Based Maintenance at Nuclear Power Plants, pp.18-55.

[5] J. T. Renwick and P. E. Babson, "Vibration analysis-a proven techniques as a productions maintenance tool," IEEE Transactions on Industry Applications, vol. 21, no. 2, pp. 324-332, 1985.

[6] R.C. Mishra, K. Pathak,_Maintenance engineering and management, pp. 42-44.

[7] M. Dileo, C.Manker, Cadick, and P. E. Jhon, "Condition based maintenance," Cadick Corporation, October 1999.

[8] L. Mann, A. Saxena, and G. M. Knapp, "Statistical-based or condition-based preventive maintenance?" Journal of Quality in Maintenance Engineering, vol. 1, no. 1, pp. 1355-2511, 1995.

[9] Richard M. Barrett Jr., Low frequency machinery monitoring: measurement consideration wilcoxon research inc, August 27, 1993.

[10] I Lasurt, AF Stronash, J Penman A fuzzy logic approach to the Interpretation of Higher order Spectra applied to fault diagnosis in electrical machine, IEEE 2000.

[ 11] Hank Lentz, Allison M. Toms, An SEM Approach to Wear Debris Analysis.

[12] DLI Engineering Corp, Integrating Vibration Analysis and Ultrasound Readings- Application Note (C) 2004, Printed in U.S.A.

[ 13] Charles W. Reeves (1998). The Vibration Monitoring Handbook, Cox moors Publishing Co. ISBN 978-1901892000.

[14] White paper, Vibration Analysis, Metso personnel, Published May $10,2011$.

[15] Simon R. W. Mills, Vibration Monitoring and Analysis Handbook (INST397), the British Institute of Non-Destructive Testing, ISBN 978090313239 7, 2010.

[ 16] Cartz, Louis, Nondestructive Testing, A $\quad$ S M International. ISBN 9780871705174, 1995.

[17] Charles Hellier, Handbook of Nondestructive Evaluation, McGrawHill, p. 1.1, ISBN 0070281211, 2003

[18] A. Korde, "On line condition monitoring of motors using electrical signature analysis," in Proceedings of the 4th International Conference on Engineering and Automation, Orlando, Florida, USA, July-August 2000.

[19] Enayet B. Halim, Sirish L. Shah, Ming J. Zuo and M. A. A. Shoukat Choudhury, Fault detection of gearbox from vibration signals using time - frequency domain averaging.
[20] Lebold, M., McClintic, K.,Campbell, R., Byington, C., and Maynard, K., Review of Vibration Analysis Methods for Gearbox Diagnostics and Prognostics, Proceedings of the 54th Meeting of the Society for Machinery Failure Prevention Technology, Virginia Beach, VA, May 1-4, 2000, p. 623-634.

[21] Eduardo Rubio and Juan C. Jáuregui, Time-Frequency Analysis for Rotor-Rubbing Diagnosis, Advances in Vibration Analysis Research.

[22] Parhi D.R.K., Behera A.K., and Behera R.K., Dynamic characteristics of cantilever beam with transverse crack. Journal of Aeronautical Society of India, Vol.47.131-143.1995.

[23] Jason R. Stack, Thomas G. Habetler, Fellow, Ronald G. Harley, Fellow, Fault-Signature Modeling and Detection of Inner-Race Bearing Faults, IEEE 2006.

[24] Bo Li,, Mo-Yuen Chow, Yodyium Tipsuwan, James C. Hung, Fellow, Neural-Network-Based Motor Rolling Bearing Fault Diagnosis, IEEE 2000.

[25] Neelam Mehala, Ratna Dahiya, Motor Current Signature Analysis and its Applications in Induction Motor Fault Diagnosis, international journal systems applications, engineering \& development Volume 2, Issue 1, 2007.

[26] D.R.K. Parhi and A.K. Dash, Faults detection by finite element analysis of a multi cracked beam using vibration signatures, Int. J. Vehicle Noise and Vibration, Vol. 6, No. 1, 2010.

[27] M.R. Nasiri, M.J. Mahjoob, H. Vahid-Alizadeh, Vibration Signature Analysis for Detecting Cavitations in Centrifugal Pumps using Neural Networks, IEEE International Conference on Mechatronics April 13-15, 2011, Istanbul, Turkey.

[28] R. K. BEHERA, D. R. K. PARHI, and S. K. SAHU, Vibration Analysis of a Cracked Rotor Surrounded by Viscous Liquid.

[29] S. Devi, Dr. L. Siva Kumar, N.R.Shanker and K.Prabakaran, A Comparative Study between Vibration and Acoustic Signals in HTC Cooling Pump and Chilling Pump, IACSIT International Journal of Engineering and Technology, Vol.2, No.3, June 2010 ISSN: 1793-8236.

[30] L. Mann, A. Saxena, and G. M. Knapp, "Statistical-based or condition-based preventive maintenance?" Journal of Quality in Maintenance Engineering, vol. 1, no. 1, pp. 1355-2511, 1995.

[31] B. Satish and D. R. Sharma, "A fuzzy BP approach for diagnostics and prognosis of bearing faults in induction motors," in IEEE Power Engineering Society General Meeting, vol. 3, pp. 22912294, San Francisco, Calif, USA, June 2005.

[32] V. Duraisamy, N. Devarajan, and D. Soma sundareswari, "Comparative study of membership functions for design of fuzzy logic fault diagnosis system for single phase induction motor," Academic Open Internet Journal, vol. 13, pp. 1-6, 2004.

[33] M. Samhouri, A. Al-Ghandoor, S. Alhaj Ali, I. Hinti, W. Massad, An Intelligent Machine Condition Monitoring System Using TimeBased Analysis: Neuro-Fuzzy Versus Neural Network, Jordan Journal of Mechanical and Industrial Engineering. All rights reserved - Volume 3, Number 4 (ISSN 1995-6665), 2009.

[34] Travers Snyman. Andrd Kel, Mechanical Condition Monitoring of Impulsively Loaded Equipment using Neural Networks, IEEE 1993.

[35] Wang Huaying Wang Guangjian, Application of Wavelet Transform and Fuzzy Theory for Turbo-Generator Fault Mode Classification, The Eighth International Conference on Electronic Measurement and Instruments ICEMI'2007.

[36] Lin Niu, A Time-delay Neural Networks Architecture for Structural Damage Detection, International Conference on Advanced Computer Control, IEEE 2008.

[37] Wang Yuguo, Xie Yan, Zhao Wei, Practical Application of Fuzzy Technology for Experiment Data Analysis of Rotating Machine, IEEE 2009

[38] L. Navarro, M. Delgado, J. Urresty, J. Cusidó, L. Romeral Condition monitoring system for characterization of electric motor ball bearings with distributed fault using fuzzy inference tools, IEEE 2010.

[39] Qun Ren, Luc Baron, Marek Balazinski, Krzysztof Jemielniak Acoustic Emission Signal Feature Analysis Using Type-2 Fuzzy Logic System, IEEE 2010.

40] Deng Lin-feng, Zhao Rong-zhen, An Improved Fuzzy Neural Network and Its Application in Machine Fault Diagnosis, International Conference on Computational and Information Sciences, IEEE 2010

[41] D.-M. Yang, Fault classification for Induction Motor using Hilbertbased Bispectral Analysis and Probabilistic Neural Networks, Eighth International Conference on Fuzzy Systems and Knowledge Discovery (FSKD), IEEE 2011 
[42] S.K. Panigrahi - S. Chakraverty - B.K. Mishra, Vibration based damage detection in a uniform strength beam using genetic algorithm Springer Science+Business Media B.V. 2009.

[43] Sun, Q., Chen, P., Zhang, D., and Xi, F., Pattern Recognition for automatic machinery faults diagnosis, Journal of Vibration and Acoustics, ASME, Vol. 126, pp-307-316, 2004.

[44] Vasile Palade, Ron J. Patton, Faisel J. Uppal, Joseba Quevedo, S. Daley, fault diagnosis of an industrialgas turbine using neuro fuzzy methods, IFAC 2002.

[45] Rafik Zouri, Sophie Sieg-Zieba, Menad Sidahmed,Fault detection system for centrifugal pumps using neural networks and fuzzy techniques.

[46] R. H. Mokhneche, H. Maaref, and V. Vigneron, Fault detection techniques analysis and development of its procedural phases.

[47] P. Jun and M. F. Lias, "A wavelet based method for extracting rolling element bearing vibration signal envelope," in Proceeding of the WSEAS International Conference on Computer Engineering and Applications, pp. 181-186, Gold Coast, Australia, January 2007.

[ 48] Pratesh Jayaswal, A. K.Wadhwani, and K. B.Mulchandani, Review Article Machine Fault Signature Analysis, International Journal of Rotating Machinery Volume 2008,

\section{BIOGRAPHIES}

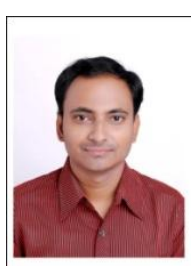

Sumit Kumar Sar pursued his Bachelor of engineering in computer science and engineering from Pt. Ravishankar Shukla University Raipur, He pursued his Masters of technology from C.S.V.T.U. Bhilai. He is an active member of ISTE. His major area of research concerned with pattern recognition and signal analysis tasks. He has various research papers in national and international conferences.

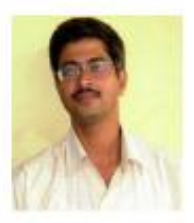

Dr. Ramesh Kumar, he received his B.E.degree in Computer Science\& Engiineering, M.Tech in Computer Science\& Engiineering,P.hD in Computer Science\& Engiineering form CSVTU Bhilai, presently working as

Professor in department of Computer Science \& Engineering, in Bhilai institute of Technology, Durg, India. 